\title{
Transient Power Flow in Micro grids with Signal Stability Energy
}

\author{
T.Yuvaraja ${ }^{1}$, Ravi.V.Angadi ${ }^{2}$ \\ ${ }^{1}$ (Department of Electrical \& Electronics Engineering, Research Scholar, Meenakshi Academy of Higher \\ Education and Research, Maher University Chennai, India) \\ ${ }^{2}$ (Department of Electrical \& Electronics Engineering, PG Scholar, JNTU University, Hyderabad, India)
}

\begin{abstract}
In micro grids the power management is obtained by energy storage. These changes are being driven from both the demand side where. higher energy availability and efficiency are desired, and from the supply side where the integration of distributed generation and peak-shaving technologies must be accommodated. Distribution systems possessing distributed generation and controllable loads with the ability to operate in both grid-connected and standalone modes are an important class of the so-called Microgrid power system. In this propose work the energy storage capacity varies and raised between the voltage limits, current limits and stability across the power limits.
\end{abstract}

Keywords: Distributed Generator(DG), transient power flow (TPG), smart grid.

\section{INTRODUCTION}

This class of Microgrid strives for optimized operation of the aggregated distribution system by coordinating the distributed generation and load resources - not only when connected to the main grid but also in a stand-alone mode. In either mode of operation, advanced local controls, energy management and protection technologies are required for robustness and reliability. While the energy management optimization objective function can be tailored to the needs of each application, in general the overall objective is to optimize operating performance and cost in the normally grid-connected mode, while ensuring that the system is capable of meeting the performance requirements in stand-alone mode. One very appealing technology for grid connected operation is tie line controls, which will regulate the active and reactive power flow between the Microgrid and the bulk grid at the point of interconnection. These controls essentially allow the Microgrid to behave as an aggregated power entity that can be made dispatch able by the utility. Particularly beneficial to the utility is the fact that this feature can be designed to compensate for intermittency associated with renewable energy resources such as wind energy and solar energy, essentially pushing the management burden inside the Microgrid. This paper reviews the overall architecture of the Microgrid concept, and presents details associated with the tie line control features.

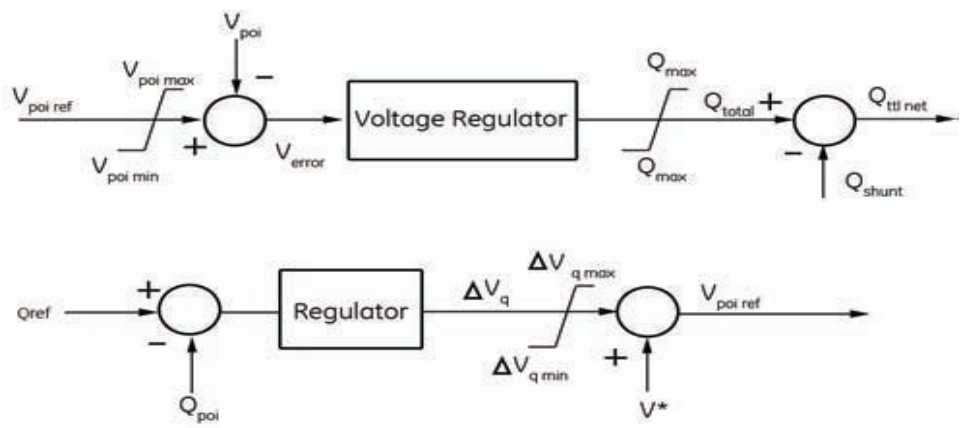

Fig 2. M-VAR Block Diagram

The feeder Microgrid will manage the generation and/or load of all entities within a distribution feeder - which can encompass 5-10MW. These Microgrids may incorporate smaller Microgrids - single or multiple facilities - within them. The appeal of these Microgrids is the potential to realize regional improvements in availability, offered by the ability of the Microgrid to separate from the bulk grid during grid disturbances and service its internal loads. Utilities, municipal utilities and coops are seen as future owners/operators of these Microgrids.

The substation Microgrid will manage the generation and/or load of all entities connected to a distribution substation - which can encompass 5-10+MW. It will likely include some generation directly at the substation, as well as distributed generation and Microgrids included at the feeder and facility level. The appeal is again the potential to realize improvements in availability, offered by the ability of the Microgrid to separate from the bulk grid during disturbances and service its internal loads. 
All of these Microgrid categories will benefit from the ability to control the dynamic exchange of power between the Microgrid and the bulk grid over the interconnecting tie line. A "tie line" refers to the feeder connection between the Microgrid and bulk grid. Tie line controls can be designed to manage the feeder power flow and voltage at the point of interconnection (POI) to meet the needs of the system operator. Control is implemented by coordinating the assets of the Microgrid, allowing the collection of these assets to appear as one aggregated dispatch able producing or consuming entity connected to the bulk grid. This section outlines the reactive and active power controls required for this capability.

\subsection{Substation Microgrids}

The primary functions of M-VAR are voltage regulation and power factor control at the tie line. Capabilities include voltage set point, steady state voltage response, and transient VAR response.

The M-VAR controller can receive either an external remote reactive power command or a voltage command from the system operator. The closed loop control issues reference VAR commands over the communication channel to each Microgrid controllable asset controller. The local controls [2] ultimately are responsible for regulating the VARs locally in each component. The controller compares the VAR output at the tie line or point of interconnection (POI) and adjusts the M-VAR command to obtain the desired system voltage. M-VAR control has two modes of operation: voltage regulated and VAR regulated (Figure 2). The voltage V poi refers to the measured line-to-line RMS value. Qpoi is to the total reactive power measured at the POI.

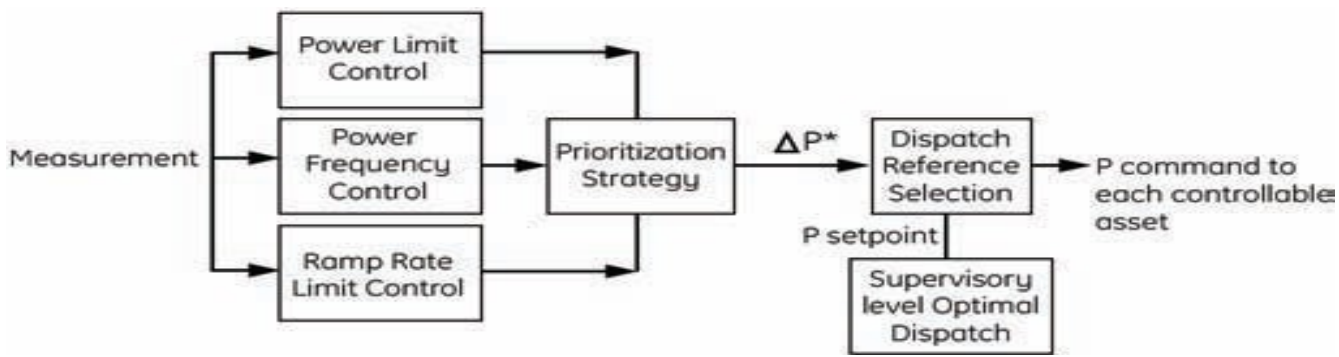

Fig. 2 M-APC Block Diagram

The error signals generated by power limit, ramp rate limit, and power frequency feed a prioritization block that selects a single error signal for control. This error signal is the input to a common M-APC regulator. The output of this regulator is a Microgrid power adjustment signal that is distributed to the controllable assets of the Microgrid.

\section{Network Topology And Power Flow Equations}

The utility grid voltage is subject to variations that are usually within $+/-5 \%$ depending mainly on the voltage level, utility system operation and design practices. The simulation shown in Figure 5 illustrates some of the performance characteristics of the M-VAR control. M-VAR has two modes of operation: voltage regulated and VAR regulated. In this case, the system was operating under voltage control. That is, the MVAR modifies reactive power of controllable sources in order to maintain the POI voltage at its set point. The test consists of a $1 \%$ voltage step change.

The active power at the POI varies due to the voltage variations inside the Microgrid. The reactive power commands to generator set 1 and the PV at the substation are modified. The response time of the system is on the order of 15 seconds. This response is relatively slow compared to typical response times of excitation controls, avoiding undesirable interactions with other controls.

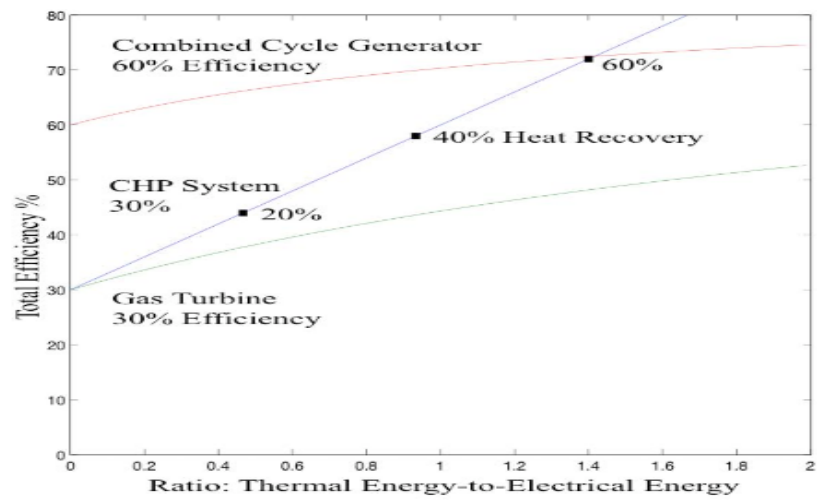




\subsection{Total System Efficiency}

Most existing power plants, central or distributed, deliver electricity to user sites at an overall fuel-toelectricity efficiency in the range of $28-32 \%$. This represents a loss of around $70 \%$ of the primary energy provided to the generator. To reduce this energy loss it is necessary to either increase the fuel-to-electricity efficiency of the generation plant and/or use the waste heat. Combined power cycles technology can attain efficiencies approaching $60 \%$ with ratings in the hundreds of million watts [1]. On the other hand if the waste heat from generators with much lower efficiency $(28-32 \%)$ can be utilized through heat exchangers, absorption chillers and/or desiccant dehumidification the overall fuel-to-useful energy efficiency can be higher than $80 \%$. Currently Capstone markets a $60 \mathrm{~kW}$ micro turbine using waste heat to heat water.

This system has a fuel to useful energy efficiency approaching $90 \%$ [2]. This use of waste heat through co-generation or combined heat and power (CHP) implies an integrated energy system, which delivers both electricity and useful heat from an energy source such as natural gas [3]. Unlike electricity, heat, usually in the form of steam or hot water, cannot be easily or economically transported long distances, so CHP systems typically provide heat for local use. Because electricity is more readily transported than heat, generation of heat close to the location of the heat load will usually make more sense than generation of heat close to the electrical load. The size of emerging generation technologies permits generators to be placed optimally in relation to heat loads. The scale of heat production for individual units is small and therefore offers greater flexibility in matching to heat requirements.

An ideal system could be constructed from the most economic combination of waste-heat-producing generators and non-waste-heat producing generators so that the combined generation of electricity and heat is optimized. In an extreme example, fuel cells could be placed on every floor of a hospital to meet each floor's hot water needs and provide electricity to local loads. For stationary energy users needing both electricity and useful heat there are two basic systems available: separate generation of electricity and heat, and combined heat and power (CHP) systems located near the heat load. To find the total energy efficiency for systems with separate generation of electricity and heat the generation efficiencies as well as the loading ratio of thermal energy-to-electrical energy needs to be known. For CHP systems the electrical and thermal recovery efficiencies along with the maximum loading level are required illustrates the total energy efficiency as a function of the loading ratio for different systems. Two systems assume separate generation of electricity and heat, the third is a CHP system. The assumed thermal generation efficiency for the value.

\subsection{Transient Power Flow With Proposed Circuit}

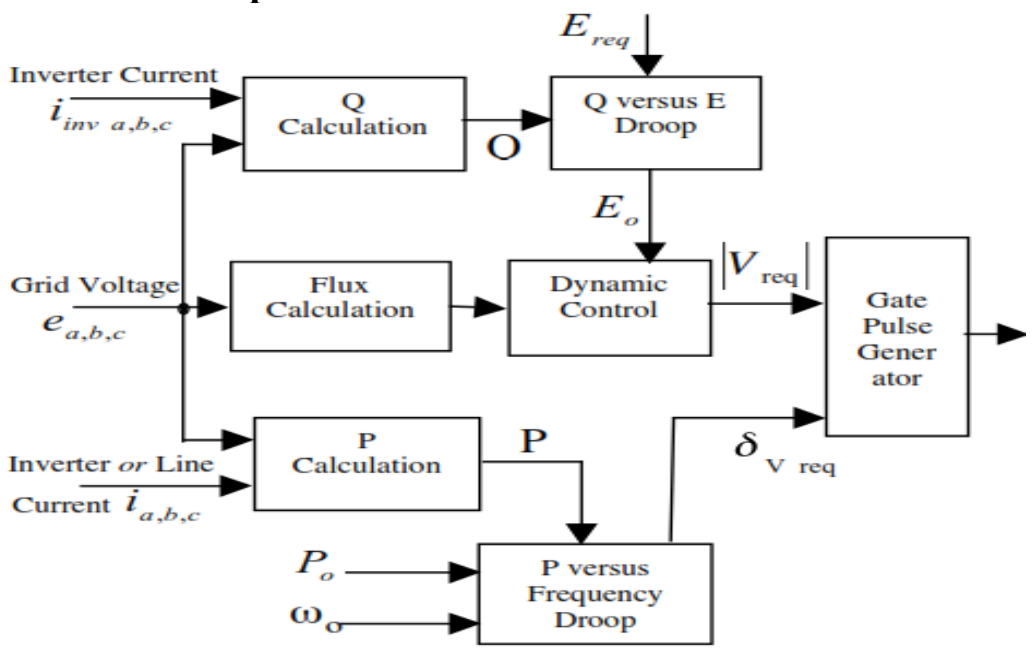

Fig. 4.Micro source power flow

\section{A.Microgrid Control}

Inverters can provide the control and flexibility required for plug-and-play functionally. Microgrid controls need to insure that; new micro sources can be added to the system without modification of existing equipment, the Microgrid can connect to or isolate itself from the grid in a rapid and seamless fashion, reactive and active power can be independently controlled, and can meet the dynamic needs of the loads Micro source controller techniques described below rely on the inverter interfaces found in fuel cells, micro turbines, and storage technologies. A key element of the control design is that communication among micro sources is unnecessary for basic Microgrid operation. Each micro source controller must be able to respond effectively to system changes without requiring data from the loads or other sources. 
Operation of the Micro grid assumes that the power electronic controls of current micro sources are modified to provide a set of key functions, which currently do not exist. These control functions include the ability to; regulate power flow on feeders; regulate the voltage at the interface of each micro source; ensure that each micro source rapidly pickups up its share of the load when the system islands.
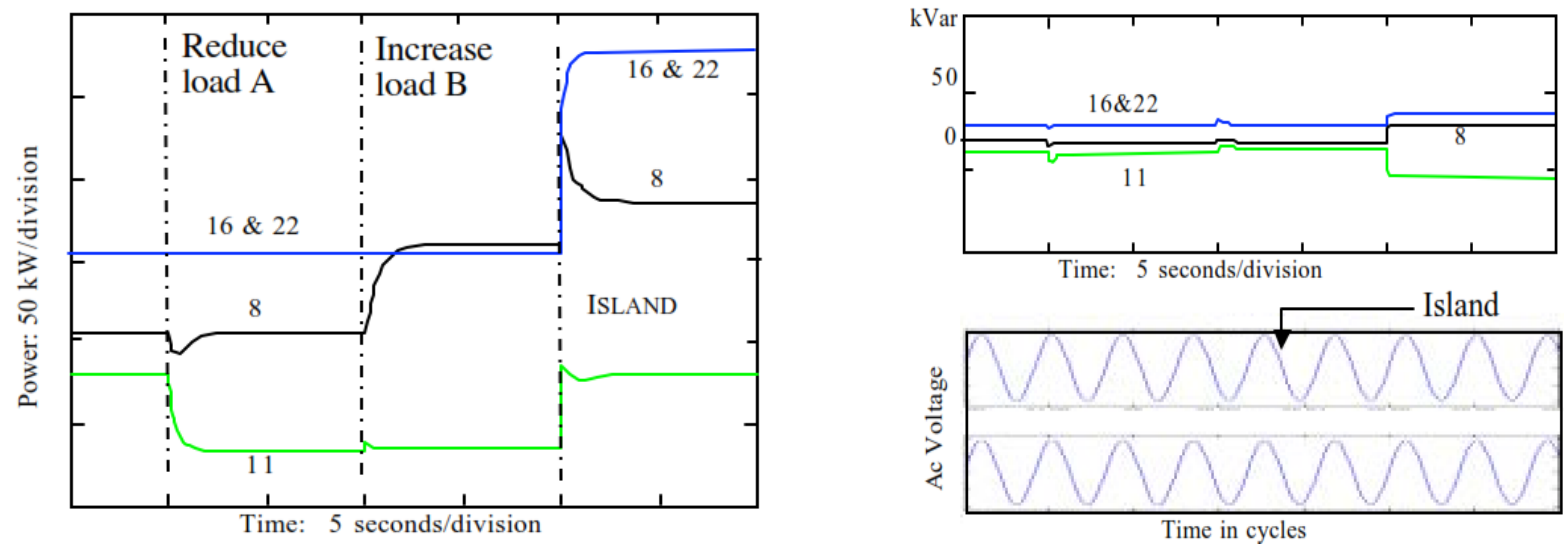

Fig. 5 and Fig. 6 Period of times cycle which a transient ratio is to be raised at stability

\section{Dynamic Process}

\section{Simulation Results}

An industrial plant with dynamic loads can be used to illustrate the dynamics of the Microgrid controls presented in the previous section, see figure 2 for a simplified layout. This industrial site has nearly $1.2 \mathrm{MWs}$ of load with motors and a $120-\mathrm{kV}$ grid supply which provides power through a long $13.8-\mathrm{kV}$ feeder consisting of overhead lines and underground cables. The plant has four main feeders at $480 \mathrm{~V}$. The loads on three $480-\mathrm{V}$ feeders are critical and must be continually served if grid power is lost. These three feeders have micro sources at nodes $8,11,16$, and 22 and the ability to island using a static switch.

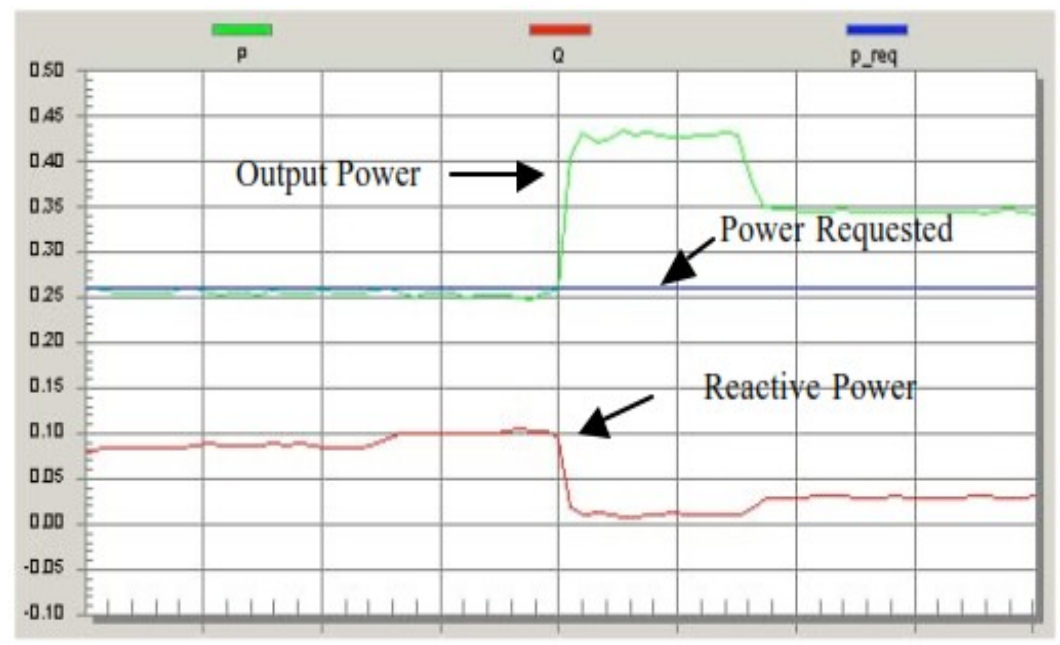

Fig.7.PQ Response of DR-8

The forth feeder stays connected to the grid. Sources at nodes 16 and 22 are on separate radial feeders while sources at nodes 8 and 11 are on the same radial feeder separated by 200 meters of cable. Load A is near node 11 while load B is between nodes 8 and 11. Figure 4 illustrates the response of the four sources to a sequence of three events; reduction of load A; followed by an increase of load B; and islanding. The "boxes" under 22, 16, 11 , and 8 also regulate the power flow in the feeder to a dispatched level. The plot on the left of figure 4 is the output power of each source for the three events. A load reduction at A results in a decrease in power flow at 8 and $11 .$. 


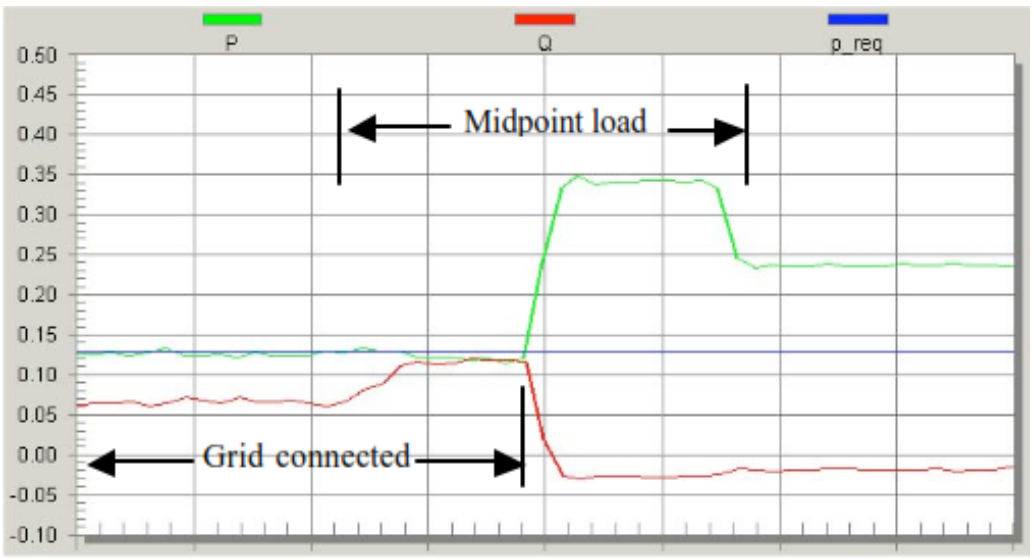

Fig.8.PQ Response of DR-11

This results in a new operating point for source 11 to hold the power flowing into feeder $\mathrm{C}$ constant. The second event is an increase in load at B. This results in an increase power output from source 8 . At the point of islanding all four sources increase their output to make up the loss of power from the grid. The top left hand plots in figure 4 are the reactive power outputs from each machine. In the absents of the voltage vs. reactive power droop these values would be more than ten times larger. The lower left curves are the voltages at 8 and 11 during loss of the utility grid.

\section{Conclusion}

The stability across the power flow is flexible at the value raised stage of BQ. The PQ response for DR- 8 and DR-11 is shown in figure 5. Figure 6 shows the currents provided by the grid and DR- 8 , the current flowing into the feeder below DR- 8 , and the voltage at the midpoint load. When the midpoint load is added figure 5 shows no change in the power output of DR8 and DR-11 with the load being supplied by the grid as shown in figure 6 . When the system islands DR-8 and DR-11 increase their power output to make up for the loss of grid power. This increase is a function of the power vs. frequency droop circuit in each controller. Also note that the reactive power is reduced since there is no longer any need to support the grid's feeder voltage. Figure 6 also demonstrates the stability of the load voltage is during these events events.

\section{References}

[1] M. Valenti "Reaching for 60 percent, the General Electric $\mathrm{H}$ turbine system taking shape in Wales is making a bid for a new record in thermal efficiency." Mechanical Engineering, April 2002.

[2] Colleen Williams, "CHP Systems, ” Distributed Energy, March/April 2004, pp.57-59.

[3] Office of Energy Efficiency and Renewable Energy, U.S. Department of Energy, "2001 BTS Core Databook," Office of Building Technology, July 13, 2001

[4] Lasseter, R., " MicroGrids, ” IEEE PES Winter Meeting, January 2002

[5] Zang, H., M.Chandorkar, G. Venkataramanan, "Development of Static Switchgear for Utility Interconnection in a Microgrid." Power and Energy Systems PES, Feb. 24-26, 2003, Palm Springs, CA

[6] Illindals, M., G. Venkataramanan, "Battery Energy Storage for StandAlone Micro-Source Distributed Generating System, " $6^{\text {th }}$ IASTED Intl. Conf. On power and Energy Systems. 2001.

[7] A. Ipakchi and F. Albuyeh, "Grid of the future," IEEE Power Energy Mag., vol. 7, n, pp. 52-62, Mar. 2009.

[8] D.Birla,R.P.Maheshwari, and H. O. Gupta, "An approach to takle the threat of sympathy trips in directional overcurrent relay coordination," IEEE Trans. Power Del., vol. 22, no. 2, pp. 851-858, Apr. 2007.

[9] D. H. Popovic,J.A.Greatbanks,M.Begovic,andA.Preglj,"Place-ment of distributed generators and reclosers for distribution network security and reliability," Int. J. Elect. Power Energy Syst., vol. 27, no. 5-6, pp. 398-408, Jun. 2005.

10] M. G. Lauby, M. Ahlstrom, D. L. Brooks, S. Beuning, J. Caspary, W.Grant, B. Kirby, M. Milligan, M.O’Malley, M. Patel, R. Piwko, P.Pourbeik, D. Shirmohammadi, and J. C. Smith, "Balancing act," IEEEPower Energy Mag., vol. 9, no. 6, pp. 75-85, Nov. 2011

[11] S. M. Brahma and A. A. Girgis, "Development of adaptive protection schemes for distribution systems with high penetration of distributed generation," IEEE Trans. Power Del., vol. 19, no. 1, pp. 56-63, Jan.2004.

[12] S. P. Chowdhury, S. Chowdhury, and P. A. Crossley, "Islanding protection of active distribution networks with renewable distributed generators: A comprehensive survey,” Elect. Power Syst. Res., vol. 79, no.6, pp. 984-992, Jun. 2009.

[13] R.Caldon,S.Andrea,andR.Turri,"Feasibility of adaptive intentional islanding operation of electric utility systems with distributed generation,"Elect. Power Syst. Res., vol. 78, no. 12, pp. 2017-2023, Dec.2008.

[14] B. Chaudhuri, A. Ghosh, R. Majumder, G. Ledwich, and F. Zare, "Improvement of stability and load sharing in an autonomous microgrid using supplementary droop control loop 2," IEEE Trans. Power Syst., vol. 25, no. 2, pp. 796-808, May 2010. 